\title{
Fractional Variational Iteration Method for Solving Fractional Partial Differential Equations with Proportional Delay
}

\author{
Brajesh Kumar Singh and Pramod Kumar \\ Department of Applied Mathematics, School for Physical Sciences, Babasaheb Bhimrao Ambedkar University, Lucknow 226 025, India \\ Correspondence should be addressed to Brajesh Kumar Singh; bksingh0584@gmail.com
}

Received 10 November 2016; Accepted 12 January 2017; Published 13 March 2017

Academic Editor: Sunil Kumar

Copyright (c) 2017 Brajesh Kumar Singh and Pramod Kumar. This is an open access article distributed under the Creative Commons Attribution License, which permits unrestricted use, distribution, and reproduction in any medium, provided the original work is properly cited.

\begin{abstract}
This paper deals with an alternative approximate analytic solution to time fractional partial differential equations (TFPDEs) with proportional delay, obtained by using fractional variational iteration method, where the fractional derivative is taken in Caputo sense. The proposed series solutions are found to converge to exact solution rapidly. To confirm the efficiency and validity of FRDTM, the computation of three test problems of TFPDEs with proportional delay was presented. The scheme seems to be very reliable, effective, and efficient powerful technique for solving various types of physical models arising in science and engineering.
\end{abstract}

\section{Introduction}

The idea of derivatives of fractional order was described first by great mathematician Newton and Leibnitz in the seventh century and has achieved a great attention due to their numerous applications in nonlinear complex systems arising in various important phenomena in the fluid mechanics, damping laws, electrical networks, signal processing, diffusion-reaction process relaxation processes, mathematical biology, and other fields of science and engineering [17]. Fractional derivatives offer more accurate models of realworld problems as compared to integer-order derivatives. The fractional calculus plays a critical role in describing a complex dynamical behavior in tremendous scope of application fields and helps to understand the nature of matter as well as simplifying the controlling design without any loss of hereditary behaviors. Further, the nonlinear oscillation of earthquake can be modeled via fractional derivatives [8]; the fluid-dynamic traffic model with fractional derivatives [9] can eliminate the deficiency arising from the assumption of continuum traffic flow and the fractional nonlinear complex model for seepage flow in porous media [10].

Indeed, it is too tough task to compute an exact solution of a wide class of the differential equations of fractional order. In the past years, different kind of vigorous techniques has been introduced to find an approximate solution of such type of fractional model of differential equations, such as generalized differential transform method [11], Adomian decomposition method [12], homotopy analysis method [13], homotopy analysis transform method [14], modified Laplace transform method [15], and homotopy perturbation transform method (HPTM) [16-18]. FRDTM have been adopted to solve vigorous types of differential equations arising in mathematics, physics, and engineering by Saravanan and Magesh [19], Srivastava et al. [20, 21], Singh and Srivastava [22], Singh and Kumar [23, 24], Singh [25], and Singh and Mahendra [26]. Recently, a fractional model of differentialdifference equation model (appeared in nanohydrodynamics, heat conduction in nanoscale, and electronic current that flows through carbon nanotubes) has been studied analytically by adopting homotopy analysis transform method [27], and a hybrid computational approach based on local fractional Sumudu transform with HPM has been employed for numerical study of Klein-Gordon equations on Cantor sets [28]. Atangana and Baleanu [4] proposed a much better version of fractional derivative with a nonsingular and nonlocal kernel, based upon the well-known generalized MittagLeffler function, to answer some outstanding questions raised 
by many researchers within the field of fractional calculus. A relationship of their derivatives with some integral transform operators was presented by Atangana and Koca [29] who show the existence and uniqueness of the system solutions of the fractional system in detail and also obtained a chaotic behavior which was not obtained by local derivative. Goufo [30] adopted newly developed Caputo-Fabrizio fractional derivative without singular kernel to obtain an analytical solution of Korteweg-de Vries-Burgers equation with two perturbations' levels. The two-parameter derivative with nonsingular and nonlocal kernel has been introduced by [31] to study the chaotic processes of the fractional system. Goufo [32] used the concept of variable-order derivative to study the stability and convergence analysis of the well-known variableorder replicator-mutator dynamics in a moving medium. For more details in fractional derivatives, we refer the readers to $[4,29-32]$ and the references therein.

The variational iteration method (VIM) has been developed by Chinese mathematician He [10]. After the seminal work of $\mathrm{He}$, various modification of VIM has been employed to solve various nonlinear problems, such as diffusion and wave equations on cantor sets [33], Riccati differential equation [34], fractional model of coupled Burgers equations [35], and time fractional Fornberg-Whitham equation [36]. For more details, the readers are referred to [33-38] and the references therein.

The partial functional differential equations with proportional delays, a special class of delay partial differential equation, arise specially in the field of biology, medicine, population ecology, control systems, and climate models [39], and complex economic macrodynamics [40].

This paper is concerned with the numerical solution of the initial valued autonomous system of TFPDEs with proportional delay [17] defined by

$$
\begin{aligned}
& \mathscr{D}_{t}^{\alpha}(u(x, t))=f\left(x, u\left(a_{0} x, b_{0} t\right), \frac{\partial}{\partial x}\right. \\
& \left.\cdot u\left(a_{1} x, b_{1} t\right), \ldots, \frac{\partial^{m}}{\partial x^{m}} u\left(a_{m} x, b_{m} t\right)\right), \\
& u^{k}(x, 0)=\psi_{k}(x),
\end{aligned}
$$

where $a_{i}, b_{i} \in(0,1)$ for all $i \in N \cup\{0\} . \psi_{k}$ is initial value, $f$ is the differential operator, and the independent variables $(x, t)$ (where $t$ denotes time and $x$ is space variable) denote the position in space or size of cells and maturation level at a time. The solution of (1) may be the voltage, temperature, densities of different particles, form instance, chemicals, cells, and so forth. One significant example of the model, Korteweg-de Vries (KdV) equation, arising in the research of shallow water waves is as follows:

$$
\mathscr{D}_{t}^{\alpha}(u(x, t))=b u \frac{\partial}{\partial x} u\left(a_{0} x, b_{0} t\right)+\frac{\partial^{3}}{\partial x^{3}} u\left(a_{1} x, b_{1} t\right)
$$

where $b$ is a constant. Another well-known model, time fractional nonlinear Klein-Gordon equation with proportional delay, aries in quantum field theory to describe nonlinear wave interaction:

$$
\begin{aligned}
\mathscr{D}_{t}^{\alpha}(u(x, t))= & u \frac{\partial^{2}}{\partial x^{2}} u\left(a_{0} x, b_{0} t\right)-b u\left(a_{1} x, b_{1} t\right) \\
& -F\left(u\left(a_{2} x, b_{2} t\right)\right)+h(x, t), \\
& 1<\alpha<2,
\end{aligned}
$$

where $b$ is a constant, $h(x, t)$ is known analytic function, and $F$ is the nonlinear operator of $u(x, t)$. For details of various types of models, we refer the reader to $[17,39]$ and the references therein.

To the best of my knowledge, there is little literature of methods to solve TFPDE with delay, such as Chebyshev pseudospectral method for linear differential and differentialfunctional parabolic equations [41], spectral collocation and waveform relaxation methods [42], and iterated pseudospectral method [43] for nonlinear delay partial differential equations. equations. Abazari and Ganji [44] obtained approximate solutions of PDEs with proportional delay by employing RDTM. Abazari and Kilicman [45] obtained analytical solutions of nonlinear integrodifferential equations with proportional delay by using DTM. Tanthanuch [46] applied group analysis method for nonhomogeneous mucilaginous Burgers equation with proportional delay. The analytical solutions of TFPDE with proportional delay have been obtained by employing homotopy perturbation method by Sakar et al. [17] and Biazar ad Ghanbari [47]. Chena and Wang [48] have adopted variational iteration method (VIM) for solving a neutral functional-differential equation with proportional delays. The main aim of this paper is to propose an alternative approximate solution of the initial valued autonomous system of TFPDE with proportional delay [17] by employing alternative variational iteration method (AVIM).

The paper is sketched into five more sections following Introduction. Specifically, Section 2 deals with the revisit of fractional calculus. Section 3 is devoted to the procedure for the implementation of the AVIM for problem (1). Section 5 is concerned with three test problems with the main aim of establishing the convergency and effectiveness of AVIM. Finally, Section 6 concludes the paper with reference to critical analysis and research perspectives.

\section{Preliminaries}

Among the various kinds of definitions of fractional derivatives, the definitions mostly applied are due to RiemannLiouville [1], Caputo [3], Yang [7], He [4], Atangana and Baleanu [4], Caputo-Fabrizio [30], and so forth. This section revisits some basic definitions of fractional calculus due to Liouville [1] which we need to complete the paper.

Definition 1. Let $\mu \in \mathbb{R}$ and $m \in \mathbb{N}$. A function $f: \mathbb{R}^{+} \rightarrow \mathbb{R}$ belongs to $\mathbb{C}_{\mu}$ if there exists $k \in \mathbb{R}, k>\mu$, and $g \in C[0, \infty)$ such that $f(x)=x^{k} g(x), \forall x \in \mathbb{R}^{+}$. Moreover, $f \in \mathbb{C}_{\mu}^{m}$ if $f^{(m)} \in \mathbb{C}_{\mu}$. 
Definition 2. Let $\mathscr{F}_{t}^{\alpha}(\alpha \geq 0)$ be Riemann-Liouville fractional integral operator and let $f \in \mathbb{C}_{\mu}$; then

$$
\begin{aligned}
(*) \mathscr{J}_{t}^{\alpha} f(t) & =(1 / \Gamma(\alpha)) \int_{0}^{t}(t-\tau)^{\alpha-1} f(\tau) d \tau, \text { if } \alpha>0, \\
(* *) \mathscr{J}_{t}^{0} f(t) & =f(t), \text { where } \Gamma(z):=\int_{0}^{\infty} e^{-t} t^{z-1} d t, z \in \mathbb{C} .
\end{aligned}
$$

For $f \in \mathbb{C}_{\mu}, \mu \geq-1, \alpha, \beta \geq 0$, and $\gamma>-1$, the operator $\mathcal{J}_{t}^{\alpha}$ satisfies the following properties:

(i) $\mathscr{J}_{t}^{\alpha} \mathscr{F}_{t}^{\beta} f(x)=\mathscr{J}_{t}^{\alpha+\beta} f(x)=J_{t}^{\beta} \mathscr{J}_{t}^{\alpha} f(x)$.

(ii) $\mathscr{J}_{t}^{\alpha} x^{\gamma}=(\Gamma(1+\gamma) / \Gamma(1+\gamma+\alpha)) x^{\alpha+\gamma}$.

It is worth mentioning that Riemann-Liouville derivative exists for any functions that are continuous but RiemannLiouville derivative has certain disadvantage for describing some natural phenomena; for example, Riemann-Liouville derivative of a constant is not equal to zero. In their work, Caputo and Mainardi [3] proposed a Caputo fractional differentiation operator $D_{t}^{\alpha}$, defined below, which is a modification of definition of Riemann-Liouville to describe the theory of viscoelasticity in order to overcome the discrepancy of Riemann-Liouville derivative [1]. It is worth mentioning that the Caputo fractional derivative allows the utilization of initial and boundary conditions involving integer-order derivatives.

Definition 3. Let $f \in \mathbb{C}_{\mu}, \mu \geq-1$, and $m-1<\alpha \leq m, m \in \mathbb{N}$. Then

$$
\begin{aligned}
\mathscr{D}_{t}^{\alpha} f(t) & =\mathscr{J}_{t}^{m-\alpha} \mathscr{D}_{t}^{m} f(t) \\
& =\frac{1}{\Gamma(m-\alpha)} \int_{0}^{t}(t-\tau)^{m-\alpha-1} f^{(m)}(\tau) d \tau .
\end{aligned}
$$

Moreover, the operator $\mathscr{D}_{t}^{\alpha}$ satisfies the following basic properties.

Lemma 4. Let $m-1<\alpha \leq m, m \in \mathbb{N}, f \in \mathbb{C}_{\mu}^{m}, \mu \geq-1$, and $\gamma>\alpha-1$, then
(a) $\mathscr{D}_{t}^{\alpha} \mathscr{D}_{t}^{\beta} f(x)=\mathscr{D}_{t}^{\alpha+\beta} f(x)$;
(b) $\mathscr{D}_{t}^{\alpha} x^{\gamma}=(\Gamma(1+\gamma) / \Gamma(1+\gamma-\alpha)) x^{\gamma-\alpha}$,
(c) $\mathscr{D}_{t}^{\alpha} \mathscr{g}_{t}^{\alpha} f(t)=f(t)$,
(d) $\mathscr{J}_{t}^{\alpha} \mathscr{D}_{t}^{\alpha} f(t)=f(t)-\sum_{k=0}^{m} f^{(k)}\left(0^{+}\right)\left(t^{k} / k !\right)$, for $t>0$.

In the present work, Caputo fractional derivative is considered as it deals with traditional initial and boundary conditions in the formulation of the physical problems. For details on fractional derivatives, we refer the interested readers to [1-7].

\section{Description of Alternative Variational Iteration Method (AVIM)}

Consider an initial valued differential equation:

$$
\begin{aligned}
L u(t)+N u(t) & =g(t), \quad t>0, \\
u^{(k)}(0) & =c_{k}, \quad k=0,1, \ldots, m-1,
\end{aligned}
$$

where $c_{k}$ are real numbers, $L=d^{n} / d t^{n}$, and $m \in \mathbb{N}$ is a linear operator; $N \rightarrow$ nonlinear operator and $g(t)$ is a known analytic function.

The correction functional for (5) can be constructed using AVIM as defined in [37] as

$$
\begin{aligned}
u_{k+1}(t)= & u_{k}(t) \\
& +\int_{0}^{t}\left[\lambda(\tau)\left(L u_{k}(\tau)-N \bar{u}_{k}(\tau)-g(\tau)\right)\right] d \tau,
\end{aligned}
$$

where the Lagrange multiplier $\lambda(\tau)$ can be identified optimally by means of variation theory. Generally, the following Lagrange multipliers are used:

$$
\lambda(\tau)=\frac{(-1)^{m}}{(m-1) !}(\tau-t)^{m-1}, \quad m \geq 1 .
$$

Equations (7) and (6) yield the following iteration formula:

$$
u_{k+1}(t)=u_{k}(t)+A\left[u_{k}(t)\right]
$$

where the operator $A[u]$ is defined by

$$
\begin{aligned}
& A(u):=\frac{(-1)^{m}}{(m-1) !} \\
& \quad \cdot \int_{0}^{t}\left((\tau-t)^{m-1}\left(L u_{k}(\tau)-N \bar{u}_{k}(\tau)-g(\tau)\right)\right) d \tau .
\end{aligned}
$$

Moreover, if we set the components $s_{k}(k=0,1,2, \ldots)$ as

$$
\begin{gathered}
s_{0}=u_{0}, \\
s_{1}=A\left[s_{0}\right], \\
s_{2}=A\left[s_{0}+s_{1}\right],
\end{gathered}
$$

$$
s_{k+1}=A\left[s_{0}+s_{1}+\cdots+s_{k}\right],
$$

then we have $u(t)=\lim _{k \rightarrow \infty} u_{k}(t)=\sum_{k=0}^{\infty} s_{k}$. Thus, (9) and (10) yield the series solution of system (5) in the following form:

$$
u(t)=\sum_{k=0}^{\infty} s_{k}(t) .
$$

The interested readers are referred to [33, 34, 36-38] for further details.

\section{AVIM for FPDEs with Proportional Delay Equations}

Consider the initial valued autonomous system of time fractional partial differential equation of order $\alpha$ with $\lceil\alpha\rceil=$ $m \in \mathbb{N}$ as

$$
\begin{aligned}
\mathscr{D}_{t}^{\alpha}\{u(x, t)\}+N u(x, t)= & g(x, t), \quad t>0, \\
u^{(k)}(x, 0)= & f_{k}(x), \\
& \quad k=0,1, \ldots, m-1, x \in R,
\end{aligned}
$$


where $N \rightarrow$ nonlinear operator, $g=g(x, t) \rightarrow$ known analytic function, and $\mathscr{D}_{t}^{\alpha} \rightarrow$ Caputo fractional derivative of order $\alpha$ and $f_{k} \rightarrow$ a real valued function.

The solution

$$
u(x, t)=\lim _{n \rightarrow \infty} u_{n}(x, t)
$$

to problem (12) can be derived from the following iteration formula as in [38]:

$$
\begin{aligned}
u_{k+1}(t)= & u_{k}(t) \\
& -J_{t}^{\alpha}\left[D_{t}^{\alpha} u_{k}(x, t)+N u_{k}(x, t)-g(x, t)\right] .
\end{aligned}
$$

The variational iteration solution, $u(x, t)=\sum_{k=0}^{\infty} v_{k}(x, t)$, in the present framework is obtained by the following iteration formula for $\lceil\alpha\rceil=m \in \mathbb{N}$ as in $[37,38]$ :

$$
\begin{aligned}
v_{0} & =\sum_{k=0}^{n-1} \frac{f_{k}(x)}{k !} t^{k}, \\
v_{k+1} & =-\frac{\Gamma(\alpha)}{\Gamma(\alpha-m+1) \Gamma(m)} \mathcal{J}_{t}^{\alpha}\left[D_{t}^{\alpha}\left[v_{0}+\cdots+v_{k}\right]\right. \\
& \left.+N\left[v_{0}+\cdots+v_{k}\right]-g(x, t)\right] .
\end{aligned}
$$

The iteration formula (15) converges to a solution of problem (12) whenever there exists $\gamma$ such that $\gamma \in(0,1)$ and $s_{k+1} \leq$ $\gamma s_{k} \forall k \in \mathbb{N} \cup\{0\}$; for details see $[37,38]$.

\section{Application of VIM to TFPDEs with Proportional Delay}

This section deals with the effectiveness and validity of VIM, which are demonstrated by means of three test problems of TFPDEs with proportional delay.

Problem 1. Consider initial values system of time fractional order, generalized Burgers equation with proportional delay as given in [17]:

$$
\begin{aligned}
\mathscr{D}_{t}^{\alpha} u(x, t)= & \frac{\partial^{2}}{\partial x^{2}} u(x, t)+u\left(\frac{x}{2}, \frac{t}{2}\right) \frac{\partial}{\partial x} u\left(x, \frac{t}{2}\right) \\
& +\frac{1}{2} u(x, t), \\
u(x, 0)= & x .
\end{aligned}
$$

Keeping (15) in mind, the iteration formula for (16) can be constructed as

$$
\begin{aligned}
s_{0}= & x \\
s_{k+1} & =-\mathscr{J}_{t}^{\alpha}\left[\mathscr{D}_{t}^{\alpha}\left\{\sum_{i=0}^{k} s_{i}(x, t)\right\}-\sum_{i=0}^{k} \frac{\partial^{2} s_{i}(x, t)}{\partial x^{2}}\right. \\
& -\frac{1}{2} \sum_{i=0}^{k} s_{i}(x, t) \\
& \left.-\left\{\sum_{i=0}^{k} s_{i}\left(\frac{x}{2}, \frac{t}{2}\right)\right\}\left\{\sum_{i=0}^{k} \frac{\partial s_{i}(x, t / 2)}{\partial x}\right\}\right] .
\end{aligned}
$$

On simplifying the above relation, we get

$$
\begin{gathered}
s_{0}=x, \\
s_{1}=\frac{t^{\alpha} x}{\Gamma[\alpha+1]}, \\
s_{2}=\frac{t^{2 \alpha} x\left(2^{1-\alpha}+1\right)}{2 \Gamma[2 \alpha+1]}, \\
s_{3}=2^{-2-3 \alpha} t^{3 \alpha} x\left[\frac{\left(2+2^{\alpha}\right)\left(2+4^{\alpha}\right)(\Gamma[1+\alpha])^{2}+2^{1+\alpha} \Gamma[1+2 \alpha]}{(\Gamma[1+\alpha])^{2} \Gamma[1+3 \alpha]}\right],
\end{gathered}
$$

The solution of problem (16) is

$$
\begin{gathered}
u(x, t)=s_{0}(x, t)+s_{1}(x, t)+s_{2}(x, t)+s_{3}(x, t)+\cdots \\
\quad=x\left\{1+\frac{t^{\alpha}}{\Gamma[\alpha+1]}+\frac{t^{2 \alpha}\left(2^{1-\alpha}+1\right)}{2 \Gamma[2 \alpha+1]}\right.
\end{gathered}
$$$$
+\left(\frac{\left(2+2^{\alpha}\right)\left(2+4^{\alpha}\right)}{2^{2+3 \alpha} \Gamma[1+3 \alpha]}+\frac{2^{-1-2 \alpha} \Gamma[1+2 \alpha]}{(\Gamma[1+\alpha])^{2} \Gamma[1+3 \alpha]}\right)
$$$$
\left.\cdot t^{3 \alpha}+\cdots\right\}
$$ 


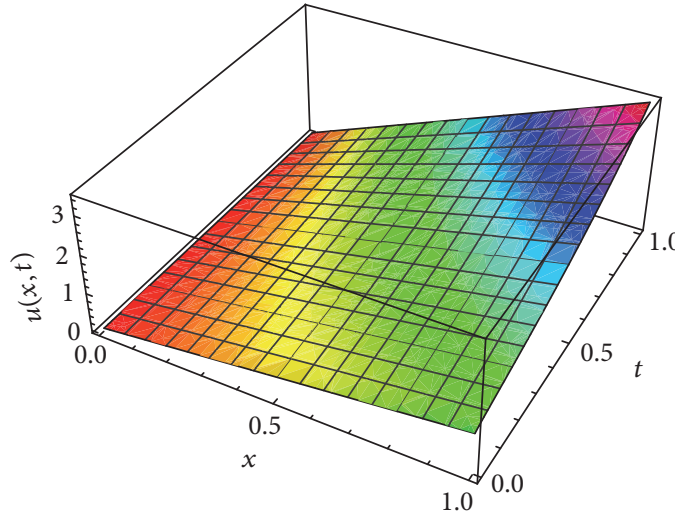

(a)

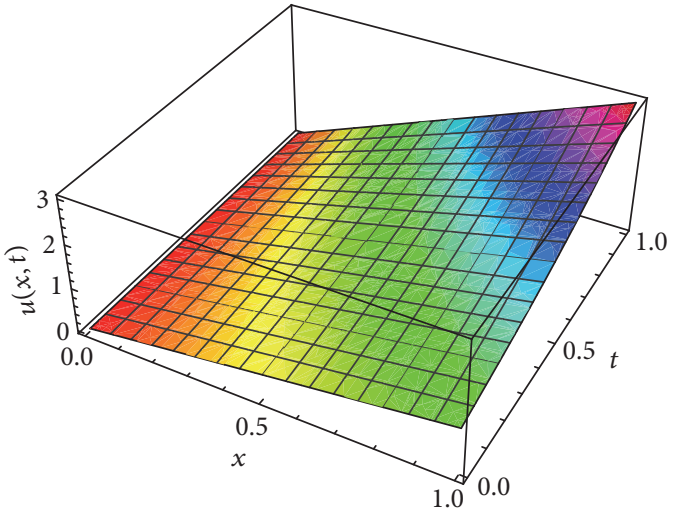

(b)

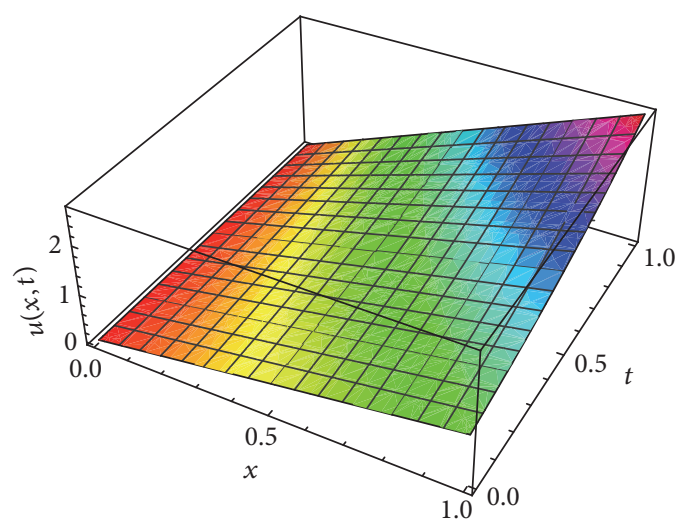

(c)

FIgURE 1: The solution behavior of AVIM solution $u$ of Example 1 for (a) $\alpha=0.8$; (b) $\alpha=0.9$; (c) $\alpha=1.0$.

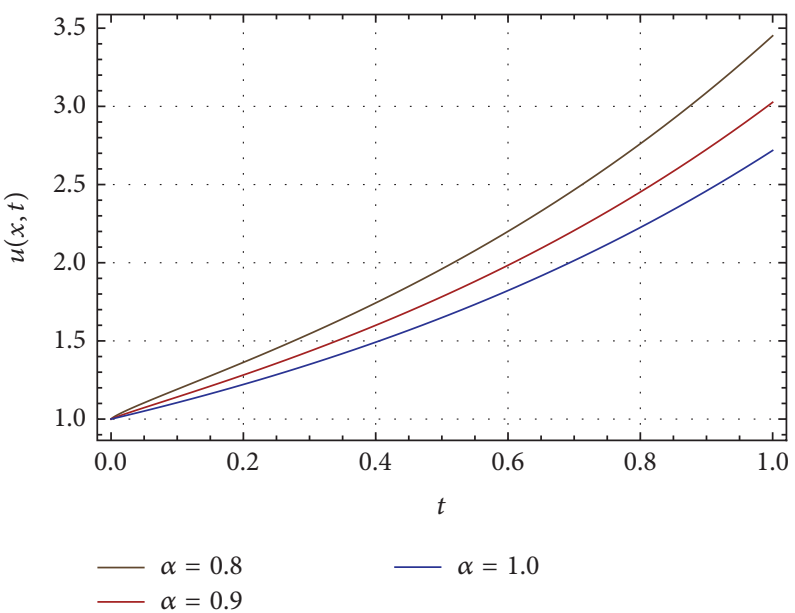

FIGURE 2: Plots of AVIM solution $u(x, t)$ of Example 1 for $\alpha=0.8,0.9,1.0 ; t \in[0,1] ; x=1$.

which is closed form to the exact solution and the results due to Sakar et al. [17] and Singh and Kumar [49]. The solution behavior of $u(x, t)$, taking first six terms, for different values of $\alpha=0.8,0.9,1.0$ at different time levels $t \leq 1$ with $x=1$, is depicted in Figure 1, whereas two dimensional plots are depicted in Figure 2.
For $\alpha=1$, solution (19) reduces to

$$
u(x, t)=x\left(1+t+\frac{t^{2}}{2 !}+\frac{t^{3}}{3 !}+\frac{t^{4}}{4 !}+\cdots\right)
$$

which is same as obtained by DTM and RDTM [44] and HPTM [49] and is a closed form of the exact solution 
$u(x, t)=x \exp (t)$. The approximate AVIM solution for $\alpha=1$ taking first six terms is reported in Table 1; it is mentioned that the results agreed well with solutions obtained by DTM and RDTM [44], HPM [17], and HPTM [49] and approach the exact solutions.

Problem 2. Consider initial value TFPDE with proportional delay as given in [17]:

$$
\begin{aligned}
\mathscr{D}_{t}^{\alpha} u(x, t) & =u\left(x, \frac{t}{2}\right) \frac{\partial^{2}}{\partial x^{2}} u\left(x, \frac{t}{2}\right)-u(x, t), \\
u(x, 0) & =x^{2}
\end{aligned}
$$

The iteration formula for (21) can be constructed as

$$
\begin{aligned}
s_{0} & =x^{2}, \\
s_{k+1} & =-\mathscr{J}_{t}^{\alpha}\left[\mathscr{D}_{t}^{\alpha}\left\{\sum_{i=0}^{k} s_{i}(x, t)\right\}+\sum_{i=0}^{k} s_{i}(x, t)\right. \\
& \left.-\left\{\sum_{i=0}^{k} s_{i}\left(x, \frac{t}{2}\right)\right\}\left\{\sum_{i=0}^{k} \frac{\partial^{2} s_{i}(x, t / 2)}{\partial x^{2}}\right\}\right] .
\end{aligned}
$$

On solving the above relation, we get

$$
\begin{gathered}
s_{0}=x^{2}, \\
s_{1}=\frac{t^{\alpha} x^{2}}{\Gamma[\alpha+1]}, \\
s_{2}=\frac{t^{2 \alpha} x^{2}\left(-2^{-\alpha}\left(-4+2^{\alpha}\right)\right)}{\Gamma[2 \alpha+1]}, \\
s_{3}=8^{-\alpha} t^{3 \alpha} x^{2}\left[\frac{\left(-4+2^{\alpha}\right)\left(-2+2^{\alpha}\right)\left(2+2^{\alpha}\right) \Gamma[1+\alpha]^{2}+2^{1+\alpha} \Gamma[1+2 \alpha]}{\Gamma[1+\alpha]^{2} \Gamma[1+3 \alpha]}\right],
\end{gathered}
$$

The solution of problem (21) leads to

$$
u(x, t)=s_{0}(x, t)+s_{1}(x, t)+s_{2}(x, t)+s_{3}(x, t)+\cdots
$$

which is closed form to the exact solution and the results due to Sakar et al. [17] and Singh and Kumar [49]. The solution behavior of $u(x, t)$ for different values of $\alpha=0.8,0.9,1.0$ at different time levels $t \leq 1$ with $x=1$ is depicted in Figure 3 , whereas two dimensional plots are depicted in Figure 4.

In particular, for $\alpha=1$, solution (24) reduces to

$$
u(x, t)=x^{2}\left(1+t+\frac{t^{2}}{2 !}+\frac{t^{3}}{3 !}+\frac{t^{4}}{4 !}+\cdots\right)
$$

which is same as obtained by DTM and RDTM [44] and HPTM [49] and is a closed form of the exact solution $u(x, t)=$ $x^{2} \exp (t)$. The approximate AVIM solution for $\alpha=1$ is reported in Table 2 . This confirms that the proposed results agreed well with solutions obtained by DTM and RDTM [44], HPM [17], and HPTM [49] and approach the exact solutions.

Problem 3. Consider initial value TFPDE with proportional delay as given in $[17,44]$ :

$$
\mathscr{D}_{t}^{\alpha} u(x, t)=\frac{\partial^{2}}{\partial x^{2}} u\left(\frac{x}{2}, \frac{t}{2}\right) \frac{\partial}{\partial x} u\left(\frac{x}{2}, \frac{t}{2}\right)
$$

$$
\begin{aligned}
& \quad-\frac{1}{8} \frac{\partial}{\partial x} u(x, t)-u(x, t), \\
& u(x, 0)=x^{2} .
\end{aligned}
$$

In particular, for $\alpha=1$, the exact solution is $u(x, t)=$ $x^{2} \exp (-t)$.

The iteration formula for (26) can be constructed as

$$
\begin{aligned}
s_{0}= & x^{2} \\
s_{k+1} & =-\mathscr{J}_{t}^{\alpha}\left[\mathscr{D}_{t}^{\alpha}\left\{\sum_{i=0}^{k} s_{i}(x, t)\right\}+\sum_{i=0}^{k} s_{i}(x, t)\right. \\
& +\frac{1}{8} \sum_{i=0}^{k} \frac{\partial s_{i}(x, t)}{\partial x} \\
& \left.-\left\{\sum_{i=0}^{k} \frac{\partial s_{i}(x / 2, t / 2)}{\partial x}\right\}\left\{\sum_{i=0}^{k} \frac{\partial^{2} s_{i}(x, t / 2)}{\partial x^{2}}\right\}\right] .
\end{aligned}
$$

Simplification of above relations leads to 


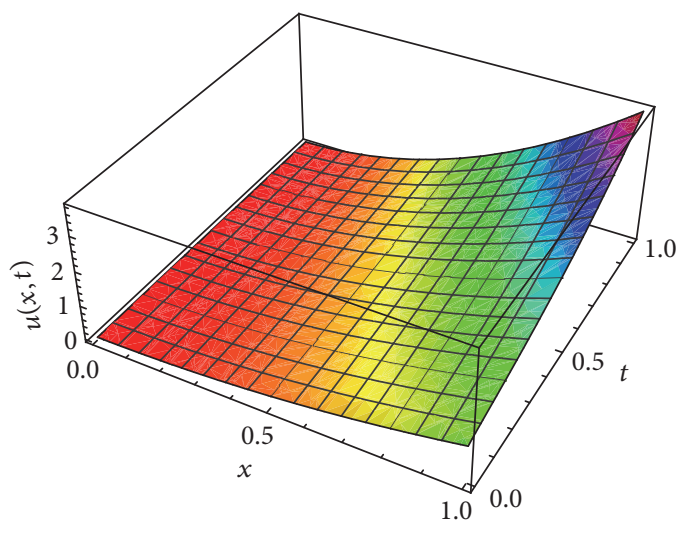

(a)

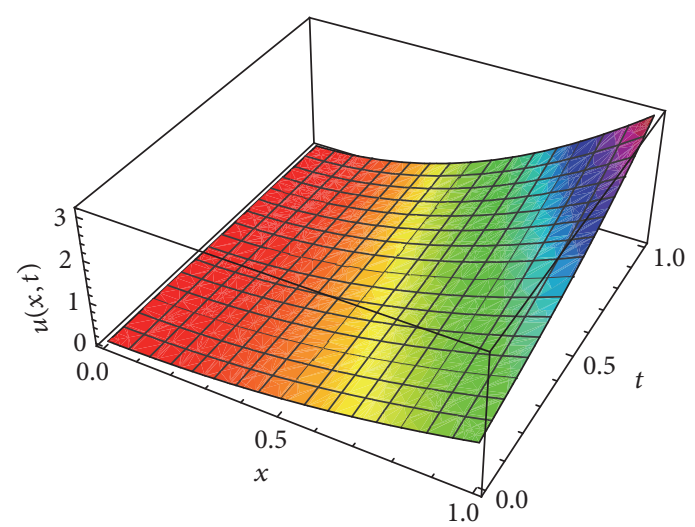

(b)

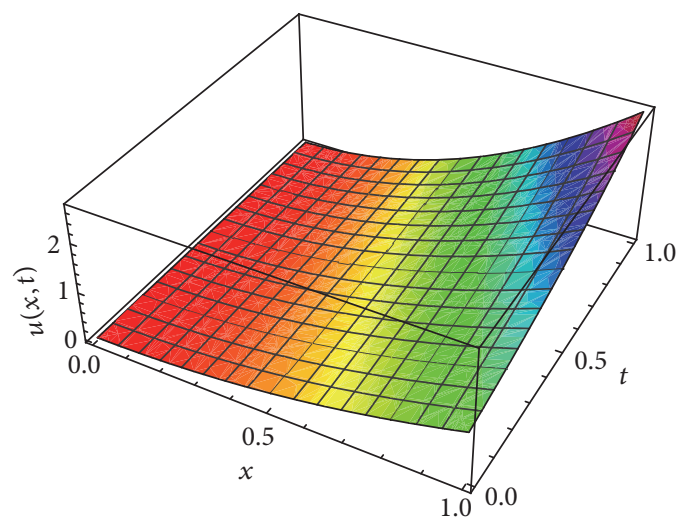

(c)

FIGURE 3: The solution behavior of AVIM solution $u$ of Example 2 for (a) $\alpha=0.8$; (b) $\alpha=0.9$; (c) $\alpha=1.0$.

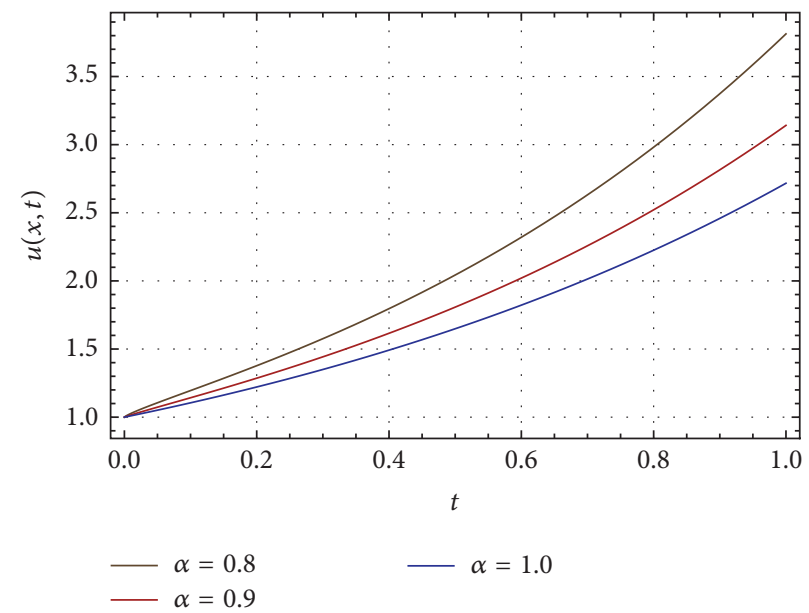

FIgUre 4: Plots of AVIM solution $u(x, t)$ of Example 2 for $\alpha=0.8,0.9,1.0 ; t \in[0,1] ; x=1$. 
TABLE 1: Approximate AVIM solution of Example 1 with first six terms for $\alpha=1$.

\begin{tabular}{|c|c|c|c|c|}
\hline \multirow{2}{*}{$x$} & \multirow{2}{*}{$t$} & \multicolumn{3}{|c|}{ VIM } \\
\hline & & Exact & Approx. & $E_{\text {abs }}$ \\
\hline \multirow{4}{*}{0.25} & 0.25 & $3.210064 E-01$ & $3.210063 E-01$ & $8.789589 E-08$ \\
\hline & 0.50 & $4.121803 E-01$ & $4.121745 E-01$ & $5.838508 E-06$ \\
\hline & 0.75 & $5.292500 E-01$ & $5.291809 E-01$ & $6.909595 E-05$ \\
\hline & 1.00 & $6.795705 E-01$ & $6.791667 E-01$ & $4.037904 E-04$ \\
\hline \multirow{4}{*}{0.5} & 0.25 & $6.420127 E-01$ & $6.420125 E-01$ & $1.757918 E-07$ \\
\hline & 0.50 & $8.243606 E-01$ & $8.243490 E-01$ & $1.167702 E-05$ \\
\hline & 0.75 & $1.058500 E+0$ & $1.058362 E+0$ & $1.381919 E-04$ \\
\hline & 1.00 & $1.359141 E+0$ & $1.358333 E+0$ & $8.075809 E-04$ \\
\hline \multirow{4}{*}{0.75} & 0.25 & $9.630191 E-01$ & $9.630188 E-01$ & $2.636877 E-07$ \\
\hline & 0.50 & $1.236541 E+0$ & $1.236523 E+0$ & $1.751553 E-05$ \\
\hline & 0.75 & $1.587750 E+0$ & $1.587543 E+0$ & $2.072879 E-04$ \\
\hline & 1.00 & $2.038711 E+0$ & $2.037500 E+0$ & $1.211371 E-03$ \\
\hline
\end{tabular}

TABLE 2: Approximate AVIM solution of Example 2 for $\alpha=1$ with first six terms.

\begin{tabular}{|c|c|c|c|c|}
\hline \multirow{2}{*}{$x$} & \multirow{2}{*}{$t$} & \multicolumn{3}{|c|}{ VIM } \\
\hline & & Exact & Approx. & $E_{\mathrm{abs}}$ \\
\hline \multirow{4}{*}{0.25} & 0.25 & $8.025159 E-02$ & $8.025157 E-02$ & $2.197397 E-08$ \\
\hline & 0.50 & $1.030451 E-01$ & $1.030436 E-01$ & $1.459627 E-06$ \\
\hline & 0.75 & $1.323125 E-01$ & $1.322952 E-01$ & $1.727399 E-05$ \\
\hline & 1.00 & $1.698926 E-01$ & $1.697917 E-01$ & $1.009476 E-04$ \\
\hline \multirow{4}{*}{0.50} & 0.25 & $3.210064 E-01$ & $3.210063 E-01$ & $8.789589 E-08$ \\
\hline & 0.50 & $4.121803 E-01$ & $4.121745 E-01$ & $5.838508 E-06$ \\
\hline & 0.75 & $5.292500 E-01$ & $5.291809 E-01$ & $6.909595 E-05$ \\
\hline & 1.00 & $6.795705 E-01$ & $6.791667 E-01$ & $4.037904 E-04$ \\
\hline \multirow{4}{*}{0.75} & 0.25 & $7.222643 E-01$ & $7.222641 E-01$ & $1.977658 E-07$ \\
\hline & 0.50 & $9.274057 E-01$ & $9.273926 E-01$ & $1.313664 E-05$ \\
\hline & 0.75 & $1.190813 E+0$ & $1.190657 E+0$ & $1.554659 E-04$ \\
\hline & 1.00 & $1.529034 E+0$ & $1.528125 E+0$ & $9.085285 E-04$ \\
\hline
\end{tabular}

$$
\begin{aligned}
& s_{0}=x^{2}, \\
& s_{1}=-\frac{t^{\alpha} x^{2}}{\Gamma[\alpha+1]}, \\
& s_{2}=\frac{2^{-2+\alpha} t^{2 \alpha} x(-2+2[\alpha](1+4 x))}{\Gamma[1+2 \alpha]}, \\
& s_{3}=\left\{\frac{8^{1+\alpha} x \Gamma[(1 / 2) \alpha]-\sqrt{\pi}\left(\left(-2+2^{\alpha}\right)\left(-2+4^{\alpha}\right)+2^{4+\alpha} x\left(-1-2^{\alpha}+4^{\alpha}(1+2 x)\right) \Gamma[1+\alpha]\right)}{2^{5+3 \alpha} \sqrt{\pi} \Gamma[1+\alpha] \Gamma[1+3 \alpha]}\right\} t^{3 \alpha},
\end{aligned}
$$

Therefore the solution for (26) is

$$
u(x, t)=s_{0}(x, t)+s_{1}(x, t)+s_{2}(x, t)+s_{3}(x, t)+\cdots
$$

which is the required solution. The similar solution behavior has been obtained in $[17,49]$. The solution behavior of $u(x, t)$ for different values of $\alpha=0.8,0.9,1.0$, at different time 


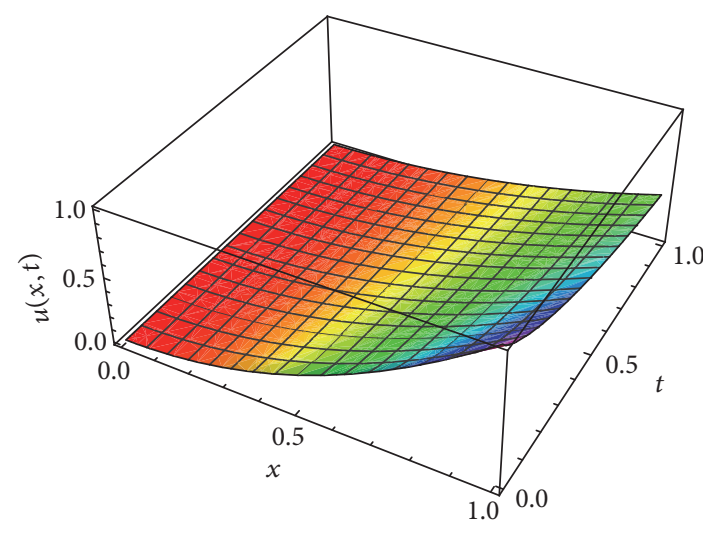

(a)

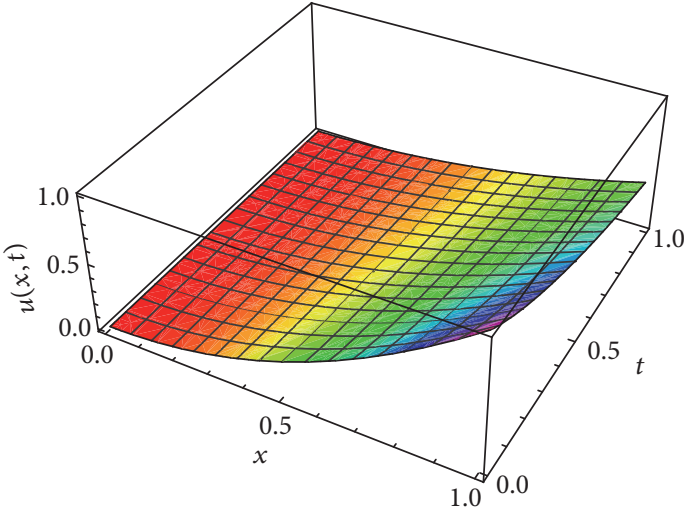

(b)

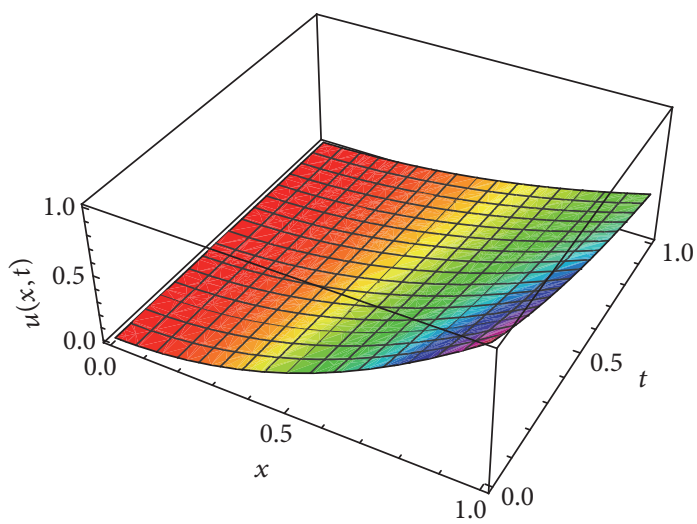

(c)

FIGURE 5: The solution behavior of VIM solution $u$ of Example 3 for (a) $\alpha=0.8$; (b) $\alpha=0.9$; (c) $\alpha=1.0$.

levels $t \leq 1$ with $x=1$, is depicted in Figure 5, whereas two dimensional plots are depicted in Figure 6. Thus, the proposed results agreed well with solutions obtained by HPM [17] and HPTM [49].

For $\alpha=1$, solution (29) reduces to

$$
\begin{aligned}
& u(x, t) \\
& \quad=\left(1-t+\frac{t^{2}}{3}-\frac{t^{3}}{6}+\frac{t^{4}}{24}-\frac{t^{5}}{120}+\frac{t^{6}}{720}-\cdots\right) x^{2}
\end{aligned}
$$

which is same as obtained by DTM and RDTM [44] and HPTM [49] and is a closed form of the exact solution $u(x, t)=$ $x^{2} \exp (-t)$. The approximate AVIM solution for $\alpha=1$ is reported in Table 3 . The proposed solution converges to the exact solution.

\section{Conclusion}

In this paper, alternative variation iteration method is successfully implemented for the numerical computation of initial valued autonomous system of time fractional model of TFPDE with proportional delay, where we use the fractional derivative in Caputo sense. The analytical results have been given in terms of a power series which converges to the exact solutions.

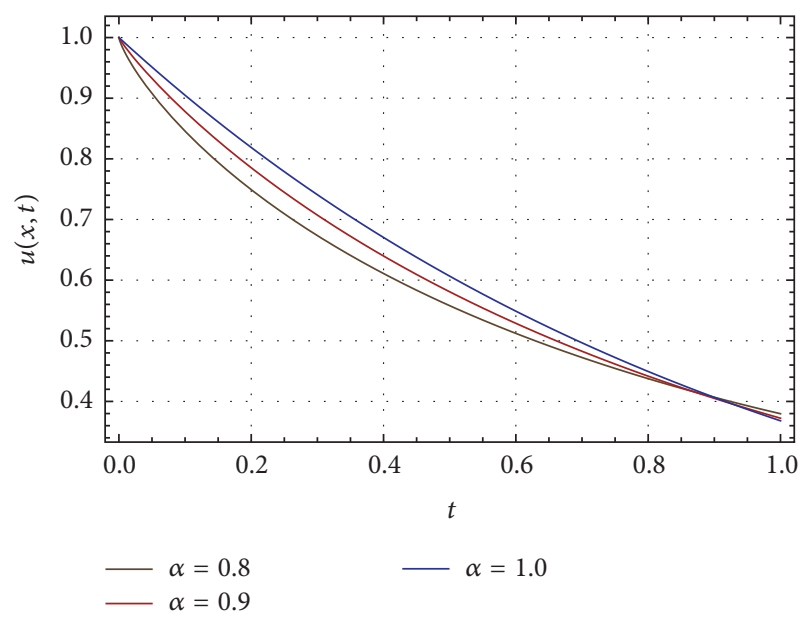

FIgURE 6: Plots of VIM solution $u(x, t)$ of Example 3 for $\alpha=0.8$, $0.9,1.0 ; t \in[0,1] ; x=1$.

Three test problems are carried out in order to validate and illustrate the efficiency of the method. The proposed solutions agreed excellently with HPM [17], HPTM [49], and DTM [44]. These approximate solutions are obtained without any discretization, perturbation, or restrictive conditions. 
TABLE 3: Approximate AVIM solution of Example 3 with first six terms for $\alpha=1$.

\begin{tabular}{ccccc}
\hline$x$ & $t$ & & VIM & $E_{\text {abs }}$ \\
& & Exact & Approx. & $2.045889 E-08$ \\
0.25 & 0.25 & $4.867505 E-02$ & $4.867503 E-02$ & $1.265190 E-06$ \\
& 0.50 & $3.790817 E-02$ & $3.790690 E-02$ & $1.393738 E-05$ \\
& 0.75 & $2.952291 E-02$ & $2.950897 E-02$ & $7.579841 E-05$ \\
\hline & 1.00 & $2.299247 E-02$ & $2.291667 E-02$ & $8.183556 E-08$ \\
0.50 & 0.25 & $1.947002 E-01$ & $1.947001 E-01$ & $5.060761 E-06$ \\
& 0.50 & $1.516327 E-01$ & $1.516276 E-01$ & $5.574951 E-05$ \\
& 0.75 & $1.180916 E-01$ & $1.180359 E-01$ & $3.031936 E-04$ \\
\hline & 1.00 & $9.196986 E-02$ & $9.166667 E-02$ & $1.841300 E-07$ \\
0.75 & 0.25 & $4.380754 E-01$ & $4.380753 E-01$ & $1.138671 E-05$ \\
& 0.50 & $3.411735 E-01$ & $3.411621 E-01$ & $1.254364 E-04$ \\
& 0.75 & $2.657062 E-01$ & $2.655807 E-01$ & $6.821857 E-04$ \\
\hline
\end{tabular}

\section{Competing Interests}

The authors declare that there are no competing interests regarding the publication of this article.

\section{Acknowledgments}

Pramod Kumar is thankful to Babasaheb Bhimrao Ambedkar University, Lucknow, India, for financial assistance to carry out the work.

\section{References}

[1] K. S. Miller and B. Ross, An Introduction to the Fractional Calculus and Fractional Differential Equations, Wiley-Interscience, New York, NY, USA, 1993.

[2] A. A. Kilbas, H. M. Srivastava, and J. J. Trujillo, Theory and Applications of Fractional Differential Equations, Elsevier, Amsterdam, The Netherlands, 2006.

[3] M. Caputo and F. Mainardi, "Linear models of dissipation in anelastic solids," La Rivista del Nuovo Cimento, vol. 1, no. 2, pp. 161-198, 1971.

[4] A. Atangana and D. Baleanu, "New fractional derivatives with nonlocal and non-singular kernel: theory and application to heat transfer model," Thermal Science, vol. 20, no. 2, pp. 763769, 2016.

[5] J.-H. He, "A tutorial review on fractal spacetime and fractional calculus," International Journal of Theoretical Physics, vol. 53, no. 11, pp. 3698-3718, 2014.

[6] E. Goldfain, "Fractional dynamics, cantorian space-time and the gauge hierarchy problem," Chaos, Solitons and Fractals, vol. 22, no. 3, pp. 513-520, 2004.

[7] X.-J. Yang, Advanced Local Fractional Calculus and its Applications, World Science, New York, NY, USA, 2012.

[8] J. H. He, "Nonlinear oscillation with fractional derivative and its applications," in Proceedings of the International Conference on Vibrating Engineering 98, vol. 9, Dalian, China, 1998.

[9] J.-H. He, "Homotopy perturbation technique," Computer Methods in Applied Mechanics and Engineering, vol. 178, no. 3-4, pp. 257-262, 1999.
[10] J.-H. He, "Approximate analytical solution for seepage flow with fractional derivatives in porous media," Computer Methods in Applied Mechanics and Engineering, vol. 167, no. 1-2, pp. 57-68, 1998.

[11] J. Liu and G. Hou, "Numerical solutions of the space- and timefractional coupled Burgers equations by generalized differential transform method," Applied Mathematics and Computation, vol. 217, no. 16, pp. 7001-7008, 2011.

[12] S. Momani and Z. Odibat, "Analytical solution of a timefractional Navier-Stokes equation by Adomian decomposition method," Applied Mathematics and Computation, vol. 177, no. 2, pp. 488-494, 2006.

[13] M. G. Sakar and F. Erdogan, “The homotopy analysis method for solving the time-fractional Fornberg-Whitham equation and comparison with Adomian's decomposition method," Applied Mathematical Modelling, vol. 37, no. 20-21, pp. 88768885, 2013.

[14] S. Kumar and D. Kumar, "Fractional modelling for BBM-Burger equation by using new homotopy analysis transform method," Journal of the Association of Arab Universities for Basic and Applied Sciences, vol. 16, pp. 16-20, 2014.

[15] S. Kumar, D. Kumar, S. Abbasbandy, and M. M. Rashidi, "Analytical solution of fractional Navier-Stokes equation by using modified Laplace decomposition method," Ain Shams Engineering Journal, vol. 5, no. 2, pp. 569-574, 2014.

[16] S. Momani, G. H. Erjaee, and M. H. Alnasr, "The modified homotopy perturbation method for solving strongly nonlinear oscillators," Computers and Mathematics with Applications, vol. 58, no. 11-12, pp. 2209-2220, 2009.

[17] M. G. Sakar, F. Uludag, and F. Erdogan, "Numerical solution of time-fractional nonlinear PDEs with proportional delays by homotopy perturbation method," Applied Mathematical Modelling, vol. 40, no. 13-14, pp. 6639-6649, 2016.

[18] J. Singh, D. Kumar, and A. Kılıçman, "Homotopy perturbation method for fractional gas dynamics equation using Sumudu transform," Abstract and Applied Analysis, vol. 2013, Article ID 934060, 8 pages, 2013.

[19] A. Saravanan and N. Magesh, "An efficient computational technique for solving the Fokker-Planck equation with space and time fractional derivatives," Journal of King Saud UniversityScience, vol. 28, no. 2, pp. 160-166, 2016. 
[20] V. K. Srivastava, N. Mishra, S. Kumar, B. K. Singh, and M. K. Awasthi, "Reduced differential transform method for solving $(1+n)$-dimensional Burgers' equation," Egyptian Journal of Basic and Applied Sciences, vol. 1, no. 2, pp. 115-119, 2014.

[21] V. K. Srivastava, S. Kumar, M. K. Awasthi, and B. K. Singh, "Two-dimensional time fractional-order biological population model and its analytical solution," Egyptian Journal of Basic and Applied Sciences, vol. 1, no. 1, pp. 71-76, 2014.

[22] B. K. Singh and V. K. Srivastava, "Approximate series solution of multi-dimensional, time fractional-order (heat like) diffusion equations using FRDTM," Royal Society Open Science, vol. 2, no. 4, Article ID 140511, 2015.

[23] B. K. Singh and P. Kumar, "FRDTM for numerical simulation of multi-dimensional, time-fractional model of Navier-Stokes equation," Ain Shams Engineering Journal, 2016.

[24] B. K. Singh and P. Kumar, "Numerical computation for timefractional gas dynamics equations by fractional reduced differential transforms method," Journal of Mathematics and System Science, vol. 6, no. 6, pp. 248-259, 2016.

[25] B. K. Singh, "Fractional reduced differential transform method for numerical computation of a system of linear and nonlinear fractional partial differential equations," International Journal of Open Problems in Computer Science and Mathematics, vol. 9, no. 3, 2016.

[26] B. K. Singh and Mahendra, "A numerical computation of a system of linear and nonlinear time dependent partial differential equations using reduced differential transform method," International Journal of Differential Equations, vol. 2016, Article ID 4275389, 8 pages, 2016.

[27] D. Kumar, J. Singh, and D. Baleanu, "Numerical computation of a fractional model of differential-difference equation," Journal of Computational and Nonlinear Dynamics, vol. 11, no. 6, Article ID 061004, 2016.

[28] D. Kumar, J. Singh, and D. Baleanu, "A hybrid computational approach for Klein-Gordon equations on Cantor sets," Nonlinear Dynamics, vol. 87, no. 1, pp. 511-517, 2017.

[29] A. Atangana and I. Koca, "Chaos in a simple nonlinear system with Atangana-Baleanu derivatives with fractional order," Chaos, Solitons \& Fractals, vol. 89, pp. 447-454, 2016.

[30] E. F. D. Goufo, "Application of the Caputo-Fabrizio fractional derivative without singular kernel to Korteweg-de VriesBurgers equation," Mathematical Modelling and Analysis, vol. 21, no. 2, pp. 188-198, 2016.

[31] E. F. D. Goufo, "Chaotic processes using the two-parameter derivative with non-singular and non-local kernel: basic theory and applications," Chaos, vol. 26, no. 8, Article ID 084305, 2016.

[32] E. F. D. Goufo, "Stability and convergence analysis of a variable order replicator-mutator process in a moving medium," Journal of Theoretical Biology, vol. 403, pp. 178-187, 2016.

[33] X.-J. Yang, D. Baleanu, Y. Khan, and S. T. Mohyud-Din, "Local fractional variational iteration method for diffusion and wave equations on Cantor sets," Romanian Journal of Physics, vol. 59, no. 1-2, pp. 36-48, 2014.

[34] F. Geng, Y. Lin, and M. Cui, "A piecewise variational iteration method for Riccati differential equations," Computers and Mathematics with Applications, vol. 58, no. 11-12, pp. 2518-2522, 2009.

[35] A. Prakash, M. Kumar, and K. K. Sharma, "Numerical method for solving fractional coupled Burgers equations," Applied Mathematics and Computation, vol. 260, pp. 314-320, 2015.
[36] M. G. Sakar and H. Ergören, "Alternative variational iteration method for solving the time-fractional Fornberg-Whitham equation," Applied Mathematical Modelling, vol. 39, no. 14, pp. 3972-3979, 2015.

[37] Z. M. Odibat, "A study on the convergence of variational iteration method," Mathematical and Computer Modelling, vol. 51, no. 9-10, pp. 1181-1192, 2010.

[38] Z. Odibat and S. Momani, “The variational iteration method: an efficient scheme for handling fractional partial differential equations in fluid mechanics," Computers \& Mathematics with Applications, vol. 58, no. 11-12, pp. 2199-2208, 2009.

[39] J. Wu, Theory and Applications of Partial Functional Differential Equations, Springer, New York, NY, USA, 1996.

[40] A. A. Keller, "Contribution of the delay differential equations to the complex economic macrodynamics," WSEAS Transactions on Systems, vol. 9, no. 4, pp. 358-371, 2010.

[41] B. Zubik-Kowal, "Chebyshev pseudospectral method and waveform relaxation for differential and differential-functional parabolic equations," Applied Numerical Mathematics, vol. 34, no. 2-3, pp. 309-328, 2000.

[42] B. Zubik-Kowal and Z. Jackiewicz, "Spectral collocation and waveform relaxation methods for nonlinear delay partial differential equations," Applied Numerical Mathematics, vol. 56, no. 3-4, pp. 433-443, 2006.

[43] J. Mead and B. Zubik-Kowal, "An iterated pseudospectral method for delay partial differential equations," Applied Numerical Mathematics, vol. 55, no. 2, pp. 227-250, 2005.

[44] R. Abazari and M. Ganji, "Extended two-dimensional DTM and its application on nonlinear PDEs with proportional delay," International Journal of Computer Mathematics, vol. 88, no. 8, pp. 1749-1762, 2011.

[45] R. Abazari and A. K1lıcman, "Application of differential transform method on nonlinear integro-differential equations with proportional delay," Neural Computing \& Applications, vol. 24, no. 2, pp. 391-397, 2014.

[46] J. Tanthanuch, "Symmetry analysis of the nonhomogeneous inviscid Burgers equation with delay," Communications in Nonlinear Science and Numerical Simulation, vol. 17, no. 12, pp. 4978-4987, 2012.

[47] J. Biazar and B. Ghanbari, “The homotopy perturbation method for solving neutral functional-differential equations with proportional delays," Journal of King Saud University-Science, vol. 24, no. 1, pp. 33-37, 2012.

[48] X. Chen and L. Wang, "The variational iteration method for solving a neutral functional-differential equation with proportional delays," Computers \& Mathematics with Applications, vol. 59, no. 8, pp. 2696-2702, 2010.

[49] B. K. Singh and P. Kumar, "Homotopy perturbation transform method for solving fractional partial differential equations with proportional delay," https://arxiv.org/abs/1611.06488v1. 


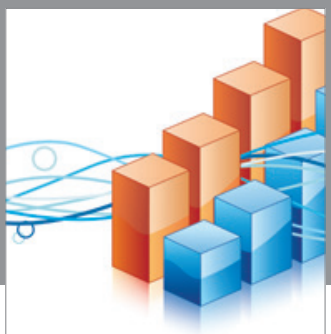

Advances in

Operations Research

vatem alat4

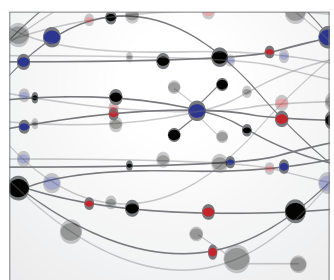

\section{The Scientific} World Journal
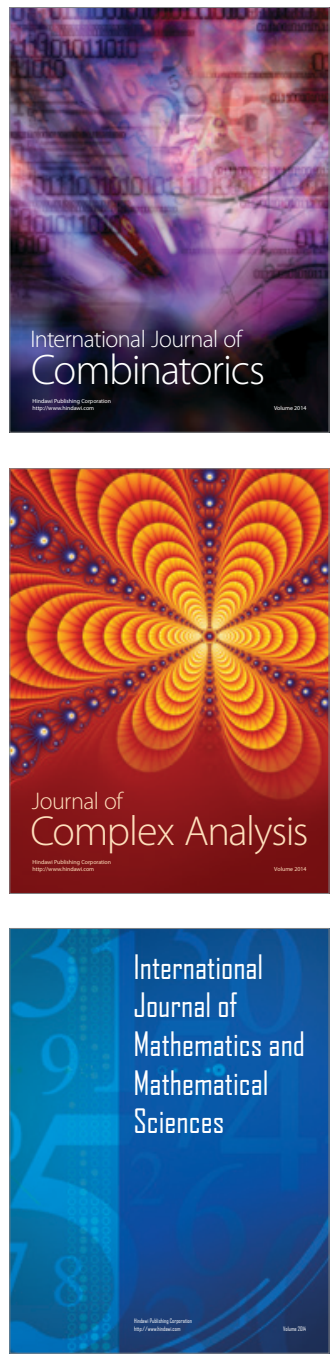
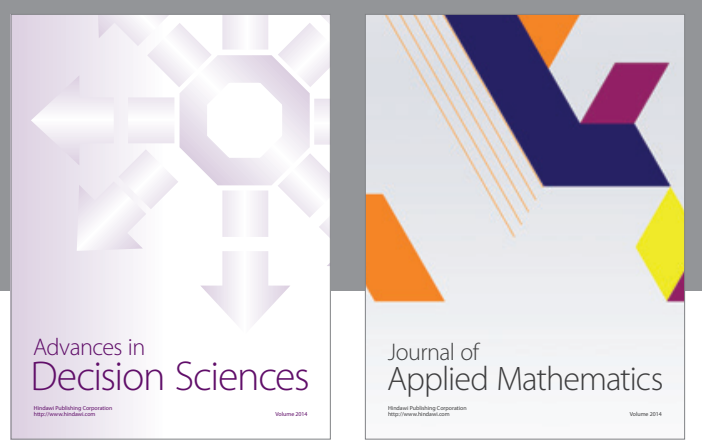

Algebra

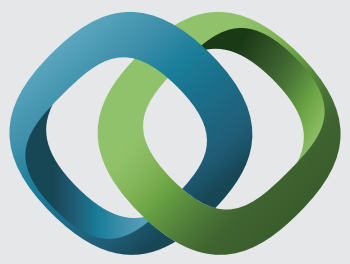

\section{Hindawi}

Submit your manuscripts at

https://www.hindawi.com
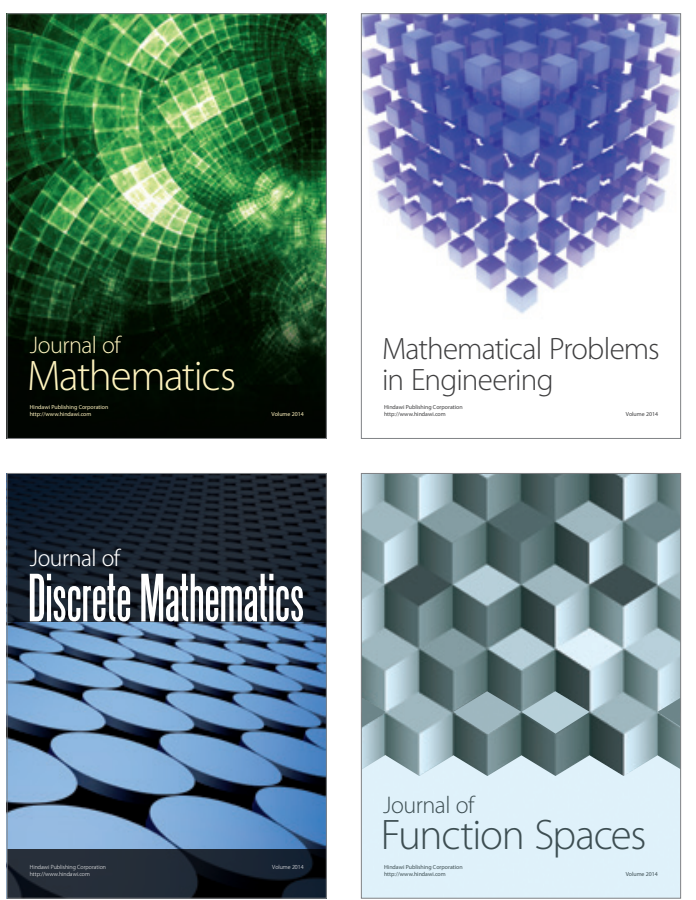

Mathematical Problems in Engineering
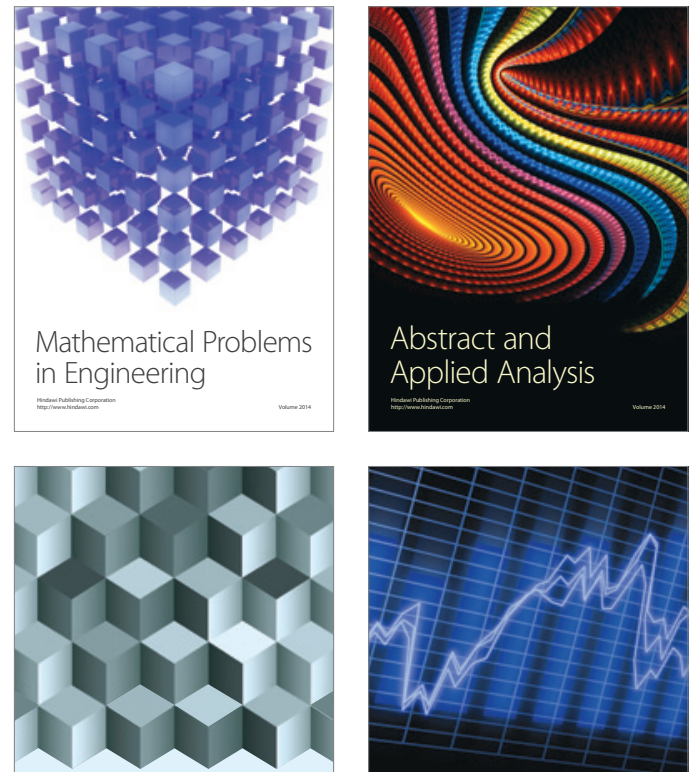

Journal of

Function Spaces

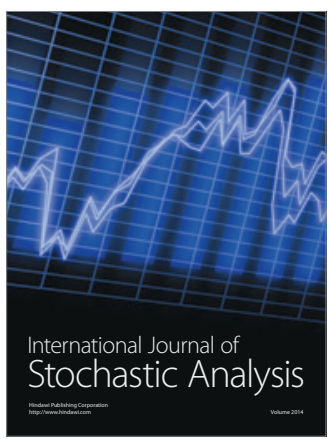

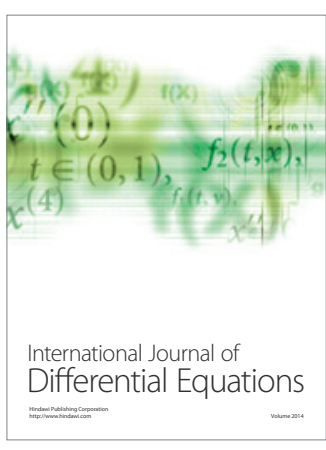
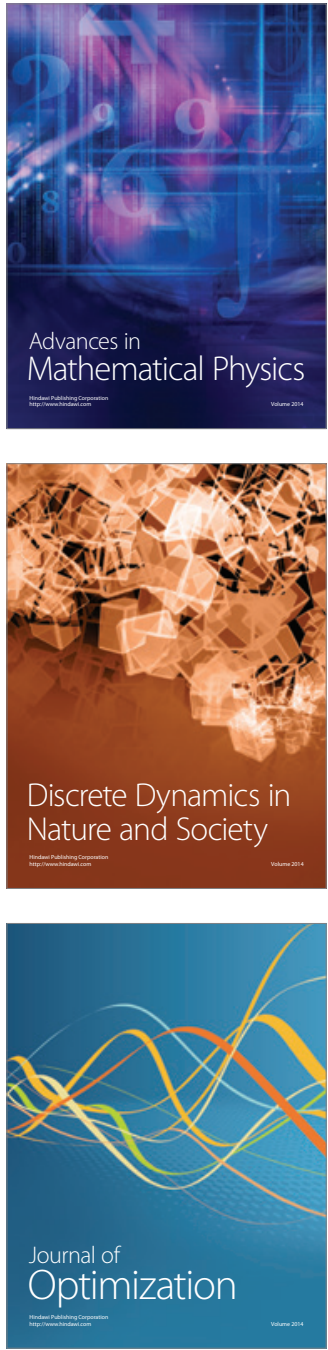